\title{
Measuring and interpreting charge dependent anisotropic flow
}

\author{
Sergei A. Voloshin*, Ronald Belmont \\ Department of Physics and Astronomy, Wayne State University, 666 W. Hancock, Detroit, Michigan 48201
}

\begin{abstract}
The Chiral Magnetic Wave (CMW) predicts a dependence of the positive and negative particle elliptic flow on the event charge asymmetry. Such a dependence has been observed by the STAR Collaboration. However, it is rather difficult to interpret the results of this measurement, as well as to perform cross-experiment comparisons, due to the dependence of the observable on experimental inefficiencies and the kinematic acceptance used to determine the net asymmetry. We propose another observable that is free from these deficiencies. It also provides possibilities for differential measurements clarifying the interpretation of the results. We use this new observable to study the effect of the local charge conservation that can mimic the effect of the CMW in charge dependent flow measurements.
\end{abstract}

Keywords: Anisotropic flow, Chiral Magnetic wave, Heavy-ion collisions

\section{Introduction}

The STAR observation [2] of the dependence of the elliptic flow of positively and negatively charged particles, $v_{2}^{+}$ and $v_{2}^{-}$on the event charge asymmetry $A_{ \pm}=\left(N_{+}-N_{-}\right) /\left(N_{+}+N_{-}\right)$(measured inside the STAR TPC acceptance) has attracted strong attention as it was found to agree, at least qualitatively, with the predictions for the Chiral Magnetic Wave (CMW) [1]. Note that more recent theoretical calculations of the CMW effect are rather controversial - they range from a very modest signal that would be difficult to detect $[3,4]$ to a "full strength" effect [5] explaining the entire signal measured by STAR. Naively, it is very difficult to imagine any background mechanisms that would lead to the dependence observed by STAR and there exist a very limited number of possible explanations proposed so far. Perhaps the most interesting of these explanations is the result of Bzdak and Bozek [6] who tried to incorporate the effect of the local charge conservation (LCC) in hydrodynamic calculations. Their calculations show that LCC can indeed be responsible for at least a large part of the observed effect. Unfortunately it is difficult to trace from the final result the real mechanism - how and why LCC actually contributes to this observable.

Note that the importance of the charge dependent anisotropic flow study goes far beyond the CMW search. In heavy-ion collisions the strength of the magnetic and electric fields can reach extremely high values $e B \sim e E \sim m_{\pi}^{2}$. The lifetime of the magnetic field is not exactly known and depends on the electric conductivity of the system. One of the recent calculations [7] shows that the lifetime of the magnetic field can be quite large, resulting in significant modifications of charged particle momentum distributions. In particular, the deflection of the charged particle trajectories in the reaction plane can contribute to the elliptic flow development. Speculating that the effect happens at the quark level it would lead to $v_{2, u}>v_{2, d} \geq v_{2, s}$. Then one should expect that $v_{2, p}>v_{2, n} \geq v_{2 . \Lambda}, v_{2, \pi^{+}}=v_{2, \pi^{-}}=v_{2, \pi^{0}}$, $v_{2, K^{+}}=v_{2, K^{-}}>v_{2, K^{0}}$. Interestingly, the recent ALICE measurements of charged and neutral kaons flow [8] would be

\footnotetext{
${ }^{*}$ Corresponding author. E-mail: sergei.voloshin@wayne.edu
} 
consistent with such relations. The precise measurements of such relations would allow us to say much more about the role of the magnetic field in the system evolution. In a very interesting scenario proposed by S. Pratt $[9,10]$ the quark production happens in two "waves" with different ratios of strange and light quarks produced at the very early times and at the hadronization stage. It would be extremely interesting to see the difference in electro-magnetic field effect on quarks produced at different times.

One of the difficulties in comparing flow results as a function of the event charge asymmetry $A_{ \pm}$obtained in different systems and/or at different collision energies is the dependence of $A_{ \pm}$on the detector acceptance as well as on experimental tracking efficiencies. The new technique discussed below is free from both of these problems, as the corresponding observable is efficiency independent and represents the dependence on the "charge asymmetry" in a differential way, allowing the comparison to be performed exactly in the (overlap) regions covered by different experiments. The differential character of the observable allows also more detailed studies of the underlying physics mechanisms. We demonstrate this below using the new observable to study LCC effects on the charge dependent anisotropic flow measurements.

\section{Correlator $\left\langle\left\langle\cos \left[n\left(\phi_{1}-\Psi_{n}\right)\right] c_{3}\right\rangle\right\rangle$}

The meaning of this correlator is the anisotropic flow of a particle as a function of the mean charge of another particle in a given momentum window. Usually we study its dependence on the pseudorapidity difference between the two particles. The notations here are the following: the particle for which the flow is measured is called particle " 1 ", the event plane is estimated with particle " 2 ", and the average charge is calculated for the particle " 3 ". Note that the average charge of particle " 3 " over the acceptance is $\left\langle c_{3}\right\rangle=A_{ \pm}$. Evaluation of the anisotropic flow is assumed to follow the standard procedure (e.g. evaluation of the event plane and subsequent correction for the reaction plane resolution, or the use of the scalar product method) and is not discussed here. The correlator is a 3-particle (point) correlator, and double brackets denote the cumulant. It assumes the subtraction of contributions from all "reducible" correlations. One has to take into account here a non-trivial character of the correlations of particle " 1 " - it is correlated with the flow angle $\Psi_{n}$ via azimuth and with particle " 3 " via both, charge and momentum.

$$
\left\langle\left\langle\cos \left[n\left(\phi_{1}-\Psi_{n}\right)\right] c_{3}\right\rangle\right\rangle=\left\langle\cos \left[n\left(\phi_{1}-\Psi_{n}\right)\right] c_{3}\right\rangle-\left\langle\cos \left[n\left(\phi_{1}-\Psi_{n}\right)\right]\right\rangle\left\langle c_{3}\right\rangle_{1}
$$

where single brackets represents the average over particles and events. $\left\langle c_{3}\right\rangle_{1}$, a function of $\Delta \eta=\eta_{1}-\eta_{3}$, is an average of the charge of particle " 3 " under condition of particle " 1 " (of a given charge) to be observed. This function is proportional to the charge balance function $B(\Delta \eta)$.
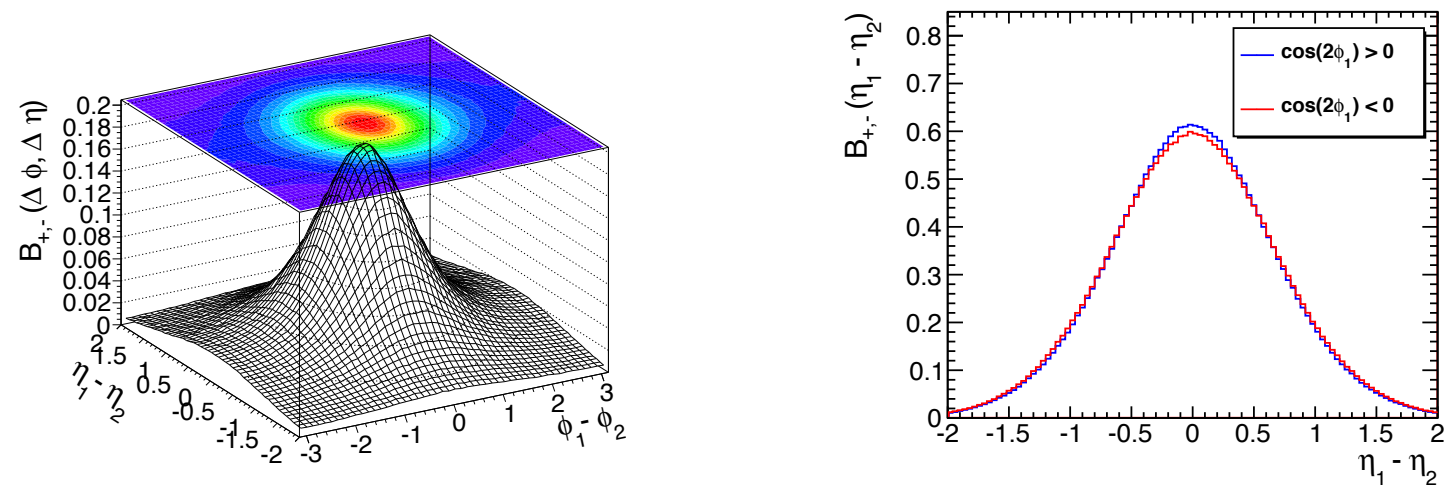

Figure 1. Left: Charge balance function as a function of $\Delta \eta, \Delta \phi$. For a description of the model see the text. Right: $\Delta \eta$ projections of the balance functions for the particle " 1 " emitted in-plane and out-of-plane. 

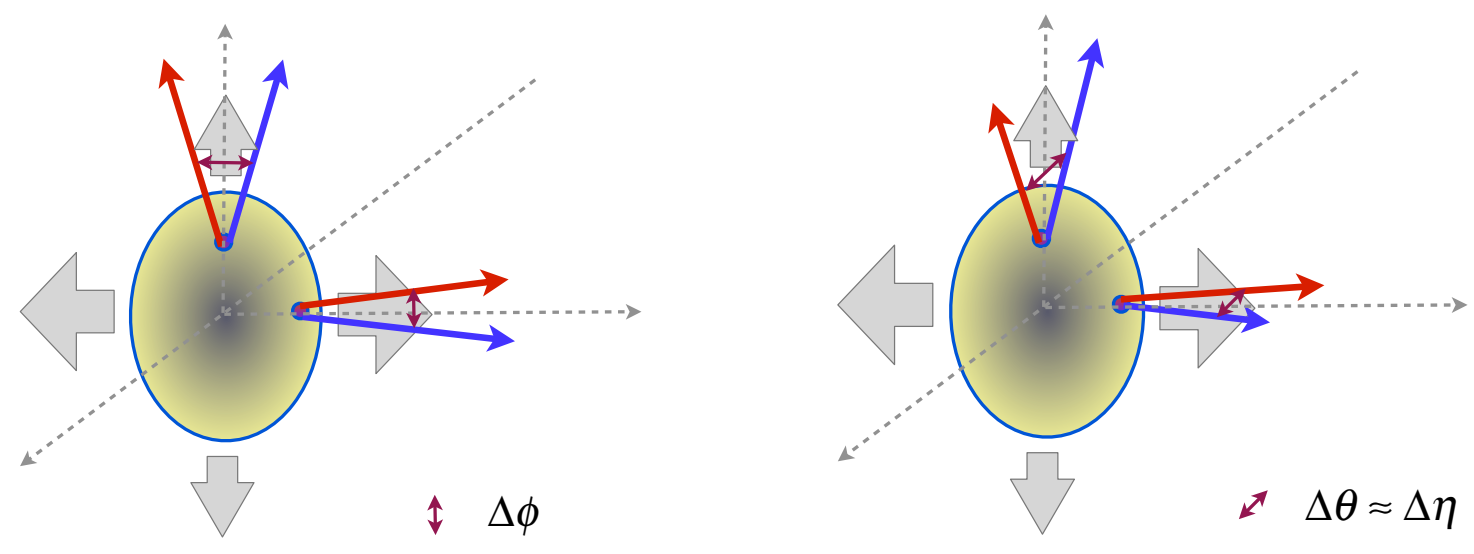

Figure 2. Effect of the radial boost on two particle angular difference in azimuth (left panel) and pseudorapidity (right panel).

\section{The Blast Wave model and effect of the local charge conservation}

To demonstrate the basic features of the proposed correlator as well as to get estimates of the LCC effect, we employ a simple Blast Wave model. The model assumes a source at constant temperature $T$, which is, with the radial boost rapidity and the number of elementary cells, $n_{c}$, modulated according to

$$
\begin{array}{r}
\rho_{t}\left(r, \phi_{b}\right)=\rho_{t, \max } \frac{r}{r_{\max }\left(\phi_{b}\right)}\left(1+\rho_{2} \cos \left[2\left(\phi_{b}-\Psi_{2}\right)\right]+\rho_{3} \cos \left[3\left(\phi_{b}-\Psi_{3}\right)\right]\right), \\
\frac{d n_{c}}{d \phi_{b}} \propto 1+s_{2} \cos \left[2\left(\phi_{b}-\Psi_{2}\right)\right]+s_{3} \cos \left[3\left(\phi_{b}-\Psi_{3}\right)\right],
\end{array}
$$

where $\phi_{b}$ is the boost angle. We assume all particles to have pion mass, and the flow angles $\Psi_{2}$ and $\Psi_{3}$ are assumed to be uncorrelated. We use $T=100 \mathrm{MeV}, \rho_{t, \max }=1.1, s_{2}=0.1, s_{3}-0.06, \rho_{2}=0.06$ and $\rho_{3}=0.03$. The parameters of the model have not been fit to the data on anisotropic flow, but selected such that they (very) roughly correspond to $\mathrm{Pb}-\mathrm{Pb}$ collisions at $\sqrt{s_{\mathrm{NN}}}=2.76 \mathrm{TeV}$ and $30 \%$ centrality. The charged particle density, also needed for correlator calculations (to account for statistical dilution of the correlations), was chosen to be $d N_{c h} / d \eta=420$.

To study the LCC effect we assume that both balancing charges are produced at the same spatial point and become correlated due to the radial (azimuthally modulated) boost according to the initial radial position. We assume that the particle production is boost invariant in the longitudinal direction, and that the particle separation in (pseudo)rapidity is due to their thermal velocities, collimated due to the radial boost [11]. The pair distribution (of the balancing charge relative to the position of the first particle) as a function of separation in pseudorapidity and azimuthal angle - the balance function - is shown in Fig. 1a. Figure 1b shows two projections of the balance function on the pseudorapidity difference for pairs selected in accordance to the emission angle of the first particle - either preferentially in-plane or out-of-plane. The widths of the two distributions are different reflecting the effect of elliptic flow, i.e. stronger radial expansion in-plane; see also Fig. 2. This effect is very similar to the contribution of LCC to the 3-particle correlator [12,13] used in search for the Chiral Magnetic Effect [14], and first discussed in [15] - stronger focusing of particle pairs emitted in-plane compared to those emitted out-of-plane.

Figure 3a presents one of the main results of this presentation - the correlator as function of the pair (particles " 1 " and "3") separation in pseudorapidity. This results is an interplay of the two effects, a stronger correlation of balancing charges in-plane compared to out-of-plane, and the statistical "dilution" of the correlation due to uncorrelated background. Note that the correlator becomes negative at $\Delta \eta \gtrsim 0.6-0.7$. It then approaches zero due to too few balancing particles at large rapidity separation. Figure $3 \mathrm{~b}$ shows the mean $p_{T}$ of the balancing charge as a function of $\Delta \eta$. The dashed line indicates the inclusive value of $\left\langle p_{T}\right\rangle$. Higher than average $p_{T}$ values are observed at small 

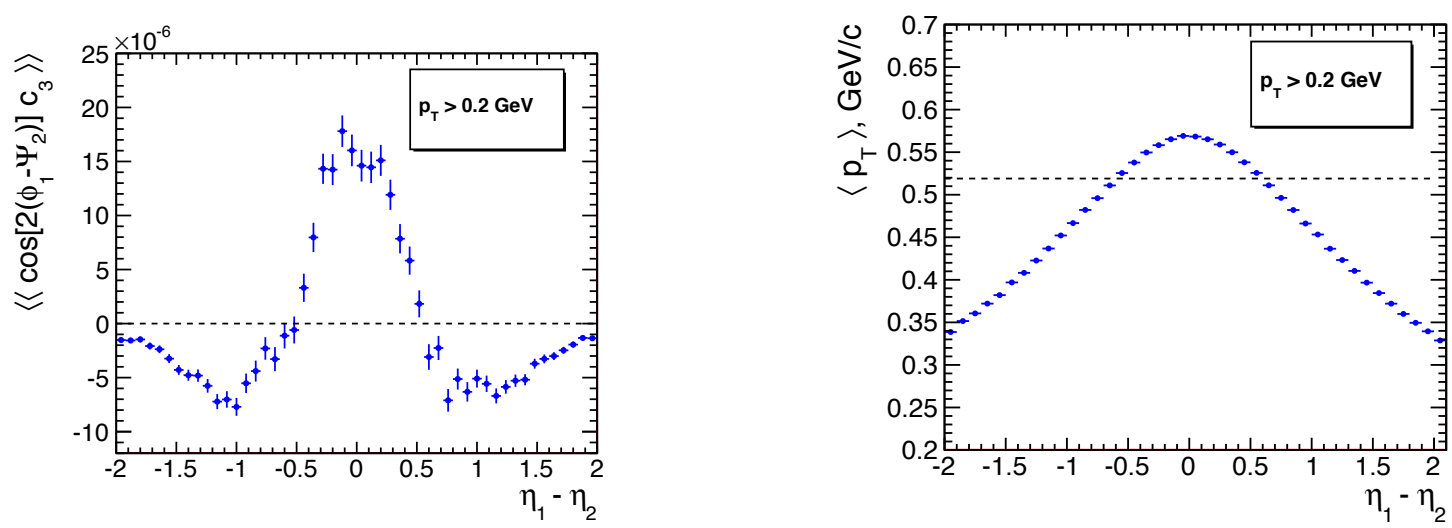

Figure 3. Left: The correlator $\left\langle\left\langle\cos \left[n\left(\phi_{1}-\Psi_{n}\right)\right] c_{3}\right\rangle\right\rangle$ as a function of $\Delta \eta$. Right: Mean $p_{T}$ of the balancing charge particle.

rapidity separation, corresponding to stronger radial flow. One can use this effect as an additional test of the effect of the LCC, e.g. by measuring $\left\langle p_{T, 3} c_{3}\right\rangle-\left\langle p_{T, 3}\right\rangle\left\langle c_{3}\right\rangle_{1}$ as a function of $\eta_{1}-\eta_{3}$.

The LCC effect should be also seen in higher harmonic correlators, while the effects of CME and CMW should be minimal [16]. According to our Blast Wave calculations the third harmonic correlator is very similar in shape to that shown in Fig. 3 with about thee times smaller an amplitude compared to the second harmonic.

\section{Conclusions}

In summary, we propose a new correlator, $\left\langle\left\langle\cos \left[n\left(\phi_{1}-\Psi_{n}\right)\right] c_{3}\right\rangle\right\rangle$, to study the charge dependence of the elliptic flow. This correlator is directly sensitive to the CMW, but, being efficiency independent and differential in nature, allows more thorough investigations of the underlying physical mechanisms. Our study of the LCC effects in the Blast Wave model reveals that stronger in-plane boost due to elliptic flow leads to narrower distribution in $\Delta \eta$ of the balancing charges, which propagates directly to the measured correlation. The results are in rough qualitative agreement with ALICE Collaboration measurements [17], but more detailed calculations, as well as measurements, are needed to draw more definite conclusions about the LCC role in a measured signal. Such Blast Wave calculations can be also used to directly relate the contribution of the LCC to the observable used for CMW search as well as the ones for the CME search, which would be an important cross check.

This material is based upon work supported by the U.S. Department of Energy Office of Science, Office of Nuclear Physics under Award Number DE-FG02-92ER-40713.

\section{References}

[1] Y. Burnier, D. E. Kharzeev, J. Liao and H. -U. Yee, Phys. Rev. Lett. 107, 052303 (2011) [arXiv:1103.1307 [hep-ph]].

[2] G. Wang [STAR Collaboration], Nucl. Phys. A 904-905, 248c (2013) [arXiv:1210.5498 [nucl-ex]].

[3] M. Hongo, Y. Hirono and T. Hirano, arXiv:1309.2823 [nucl-th].

[4] S. F. Taghavi and U. A. Wiedemann, arXiv:1310.0193 [hep-ph].

[5] H. -U. Yee and Y. Yin, Phys. Rev. C 89, 044909 (2014) [arXiv:1311.2574 [nucl-th]].

[6] A. Bzdak and P. Bozek, Phys. Lett. B 726, 239 (2013) [arXiv:1303.1138 [nucl-th]].

[7] U. Gursoy, D. Kharzeev and K. Rajagopal, Phys. Rev. C 89, 054905 (2014) [arXiv:1401.3805 [hep-ph]].

[8] B. B. Abelev et al. [ALICE Collaboration], arXiv:1405.4632 [nucl-ex].

[9] S. Pratt, Phys. Rev. Lett. 108, 212301 (2012) [arXiv:1203.4578 [nucl-th]].

[10] S. Pratt, PoS CPOD 2013, 023 (2013) [arXiv:1304.2442 [nucl-th]].

[11] S. A. Voloshin, Phys. Lett. B 632, 490 (2006) [nucl-th/0312065].

[12] S. A. Voloshin, Phys. Rev. C 70, 057901 (2004) [hep-ph/0406311].

[13] B. I. Abelev et al. [STAR Collaboration], Phys. Rev. Lett. 103, 251601 (2009) [arXiv:0909.1739 [nucl-ex]].

[14] D. E. Kharzeev, L. D. McLerran and H. J. Warringa, Nucl. Phys. A 803, 227 (2008) [arXiv:0711.0950 [hep-ph]].

[15] S. Schlichting and S. Pratt, Phys. Rev. C 83, 014913 (2011) [arXiv:1009.4283 [nucl-th]].

[16] S. A. Voloshin, Prog. Part. Nucl. Phys. 67, 541 (2012) [arXiv:1111.7241 [nucl-ex]].

[17] R. Belmont for the ALICE Collaboration, these proceedings. 